\title{
The risk of using monoclonal or polyclonal commercial antibodies: controversial results on porcine sperm CD44 receptor identification
}

Manuel Alvarez-Rodriguez, Manel Lopez-Bejar and Heriberto Rodriguez-Martinez

The self-archived postprint version of this journal article is available at Linköping University Institutional Repository (DiVA):

http://urn.kb.se/resolve?urn=urn:nbn:se:liu:diva-157233

N.B.: When citing this work, cite the original publication.

Alvarez-Rodriguez, M., Lopez-Bejar, M., Rodriguez-Martinez, H., (2019), The risk of using monoclonal or polyclonal commercial antibodies: controversial results on porcine sperm CD44 receptor

identification, Reproduction in domestic animals, 54(4), 733-737. https://doi.org/10.1111/rda.13415

Original publication available at:

https://doi.org/10.1111/rda.13415

Copyright: Wiley (12 months)

http://eu.wiley.com/WileyCDA/ 


\title{
The risk of using monoclonal or polyclonal commercial antibodies: controversial results on porcine sperm CD44 receptor identification.
}

Alvarez-Rodriguez, Manuel ${ }^{1,2 *}$, López-Bejar, Manel$^{2}$, Rodriguez-Martinez, Heriberto ${ }^{1}$

${ }^{1}$ Department of Clinical and Experimental Medicine (IKE), BKH, Obstetrics and Gynaecology, Faculty of Medicine \& Health Sciences, Linköping University, Linköping, Sweden

${ }^{2}$ Department of Animal Health and Anatomy, Veterinary Faculty, Universitat Autònoma de Barcelona, Bellaterra, Spain.

Correspondence: Manuel Álvarez-Rodriguez

Faculty of Medicine and Health Sciences, Department of Clinical and Experimental Medicine (IKE), Obstetrics and Gynaecology, Linköping University, SE-58115, Linköping, Sweden

E-mail: manuel.alvarez-rodriguez@liu.se/manualvro@gmail.com

Running title: Specific detection of CD44 receptor in boar sperm.

\begin{abstract}
Presence of the hyaluronan (hyaluronic acid, HA) receptor CD44 on spermatozoa has been difficult to pursue, mostly obeying to the use of different commercial mono- and/or polyclonal antibodies, often lacking proper controls. Here, we describe how the presence (Western blotting) and specific location (immunocytochemistry) of the CD44 receptor differs in ejaculated pig spermatozoa depending on the type of antibody and protocol used. While we were able to detect binding to spermatozoa and mark its presence in the sperm membrane, the use of blocking peptides clearly indicated that only the monoclonal antibody could confirm the specific presence and location of the CD44 receptor, whereas the polyclonal antibody was detecting multiple presumed CD44 isoforms or degraded proteins thus proving unspecific. These results call for strict protocols when attempting immunological determination of sperm membrane receptors.
\end{abstract}


Keywords: CD44 receptor, Hyaluronan, spermatozoa, polyclonal antibody, monoclonal antibody, pig.

\section{Introduction}

Hyaluronan (hyaluronic acid, HA) is a ubiquitous non-sulphated glycosaminoglycan (GAG) built by an unbranched linear chain of repeating N-acetyl-D-glucosamine and D-glucuronic acid disaccharide units, which displays a surprising wide range of structural, physiological, and pathological roles, creating and filling up extracellular matrixes (see recent review by Rodriguez-Martinez et al. (2016)). Hyaluronan modulates cell responses by acting as a ligand to specific HA membrane receptors (Thorne et al., 2004), among which the transmembrane CD44 receptor is the most commonly recognized (Day et al., 2002). The CD44 receptor is present in the pig pre-ovulatory oviductal sperm reservoir (Tienthai et al., 2003) as well as in mural and cumulus granulosa cells (Yokoo et al., 2002) presumably acting as adhesion receptor for cell attachment on an HA substratum and as a mediator of the transduction of intracellular signals leading to changes in cell proliferation, survival, and differentiation within the HA-turnover system (Toole et al., 2004; Tienthai, 2015). Spermatozoa from several species seem to depict a functionally active, HA-adhesive form of the CD44 receptor in their plasma membrane where HA would bind (Rodriguez-Martinez et al., 2016) including boar spermatozoa (Alvarez-Rodriguez et el., 2018), although sperm HA-exposure does not always elicit functional responses (Alvarez-Rodriguez et el., 2018). Such findings prompted critical comparisons between monoclonal and polyclonal antibodies, commercially available, blocked with immunogene peptides to determine the specificity of the CD44 receptor in ejaculated and extended for liquid-storage pig spermatozoa.

\section{Materials \& methods}


All reagents were obtained from Sigma-Aldrich (Sweden), unless otherwise stated. Primary antibodies and blocking peptide for WB and ICC were purchased from Abcam (Cambridge, UK) or Nordic BioSite (Proteintech Europe; Manchesterm, UK), while secondary antibodies were purchased from Abcam (Cambridge, UK), LI-COR Biosciences (Lincoln, NE, USA) or Invitrogen (Carlsbad, CA, USA).

Ejaculated pig spermatozoa were obtained as commercial AI doses from Köttböndernas, (Hållsta, Sweden), extended to $2.5 \times 10^{9}$ total spermatozoa/dose. Each dose batch was built by pooling ejaculates from 3 boars of proven fertility, extended in Durasperm ${ }^{\mathrm{TM}}$ [Jørgen Kruuse $\mathrm{A} / \mathrm{S}$, Langeskov, Denmark]), and stored at the $17-20^{\circ} \mathrm{C}$ temperature range. All experiments were performed at the Department of Clinical and Experimental Medicine, Linköping University, Linköping, Sweden, in accordance with the relevant animal research regulations of the European Union (EC-Directive 2010/63/EU) and in compliance with current Swedish legislation (SJVFS 2017:40). The experimental protocol, including postmortem collection of pig vesicular glands (to isolate proteins to be used as positive somatic control, Castellà Fernández, 2000), had been previously reviewed and approved by the Local Ethical Committee for Experimentation with Animals at Linköping, Sweden (permit nr 74-12 and nr ID-1400). Ejaculated human spermatozoa provided by anonymous, fertile donors who gave written consent were subjected to gradient density cleansing (PureSperm, Nidacon, Göteborg, Sweden), at the Reproductive Medicine Center (RMC), Region Östergötland, Linköping, Sweden (Ethical Permission Number Dnr 2015/387-31) and were used as CD44 receptor positive sperm control.

Western blotting was performed following the described protocol by Alvarez-Rodriguez et al. (2018). Briefly, after total protein extraction and quantification, $2.5 \mu \mathrm{g}$ protein of each protein suspension were loaded into NuPAGE 4-12\% Bis-Tris SDS-PAGE gels (Life Technologies, 
Carlsbad, CA, USA) and transferred to polyvinyldifluoride (PVDF) membranes (Invitrolon PVDF filter paper sandwich, Life Technologies). Then, blocked at room temperature for 60 min with Odyssey blocking buffer solution (OBBF; LI-COR Biosciences, Lincoln, NE, USA). After extensive washing in PBST (Phosphate-buffered saline (PBS); ThermoFisher Scientific, Waltham, MA, USA) containing 0.1\% Tween-20 (Sigma-Aldrich) (PBST), membrane was incubated at $4{ }^{\circ} \mathrm{C}$ overnight with the primary polyclonal antibody $(1: 1,000$ dilution, anti-CD44 antibody ab24504; Rabbit polyclonal to CD44; Abcam, Cambridge, UK) or with the primary monoclonal (1:500 dilution, anti-CD44 antibody 60224-1-Ig; mouse monoclonal antibody to CD44; species specificity: pig; Nordic BioSite, Proteintech Europe, Manchester, UK). The specificity of the polyclonal antibody was tested by incubation of the primary antibody in presence of its specific blocking peptide (ab52122 Human CD44 peptide; Abcam, Cambridge, UK) at 1:5 ratio. The specificity of the monoclonal antibody was previously tested by Alvarez-Rodriguez et al. (2018). After overnight incubation, the membranes were washed in PBST and incubated for 60 min with a dilution 1:15,000 of the secondary antibody for the polyclonal primary antibody (anti-rabbit IRDye $800 \mathrm{CW}$ (925-32213, Abcam, Cambridge, UK) or for the monoclonal primary antibody (goat anti-mouse IRDye $800 \mathrm{CW}$ (925-32210, LI-COR Biosciences, Lincoln, NE, USA). Then, after extensive washing in PBST, the membranes were scanned using the Odyssey CLx (LI-COR Biosciences, Lincoln, NE, USA), and images of the blots were obtained using the Image Studio 4.0 software (LI-COR Biosciences, Lincoln, NE, USA).

Immunocytochemistry was performed following the described protocol by Alvarez-Rodriguez et al. (2018). Human (positive control) and porcine spermatozoa were fixed in $4 \%$ paraformaldehyde at room temperature (r.t.) for $20 \mathrm{~min}$. After centrifugation, the pellet was resuspended in PBS and prepared in smears on poly-L-lysine slides (LSM, Thermo Scientific, 
Germany). The smears were dried at r.t., washed with PBS, and blocked with $\mathrm{OBBF}$ at $4^{\circ} \mathrm{C}$ for $120 \mathrm{~min}$. After further washing with PBST, the slides were incubated at $4^{\circ} \mathrm{C}$ overnight with the primary polyclonal or monoclonal antibody (see WB protocol) diluted 1:100 in OBBF. Then, the slides were incubated with the secondary antibody in the dark at room temperature for 75 min with a 1:1,000 dilution of Alexa Fluor 568-conjugated goat anti-rabbit antibody (Invitrogen, Carlsbad, CA, USA) or 1:1,000 dilution of Alexa Fluor 488-conjugated goat anti-mouse IgG H\&L (ab150113, Abcam, Cambridge, UK) in OBBF. The smears were mounted with Prolong Gold anti-fade reagent (Invitrogen, Carlsbad, CA, USA). Negative controls were performed using the blocking peptide (ab52122 Human CD44 peptide; Abcam, Cambridge, UK) (see WB protocol). Results were collected using a Zeiss Axioscop microscope (Zeiss, Germany) equipped with an epifluorescence module and a CCD Camera (QIMAGING, Canada) at 1,000× magnification. At least 200 cells were counted per replicate. Immunolabeling was present in $>95 \%$ of the spermatozoa and absent in the negative controls.

\section{Results}

The WB-analysis was performed using two different types of antibodies, a non-specific polyclonal antibody (Fig 1, Gel A) and a specific porcine monoclonal anti-CD44 antibody (Fig 1, Gel C). Blocking with the peptides used as immunogens for both the monoclonal (Fig 1, Gel D) and polyclonal antibodies were used (Fig 1, Gel B). The WB analysis intended the specific detection of the CD44 receptor in human (Lanes 2) and porcine spermatozoa (Lanes 3), and a somatic cell control (vesicular glands, Lanes 4) (Fig 1, Gels A-C), comparing antibodies. In human spermatozoa (positive control), the CD44 appeared as a single band of approx. $25 \mathrm{kDa}$ (Fig 1, Gel A, Lane 2) while pig spermatozoa and showed two main bands of approximately 50 and $40 \mathrm{kDa}$ (Fig 1, Gel A, Lanes 3). Co-incubation of the polyclonal primary antibody with its blocking peptide was only able to block a few CD44 presumed 
isoform bands either completely (arrow in Fig 1, Gel A, Lane 2) or incompletely (Fig 1, Gel B, Lanes 3-4) indicating most bands detected in A were unspecific or CD44 isoforms. The major band CD-44 in somatic cells (boar vesicular glands, Fig 1, Gel A-C, Lane 4) was consistently present at approx. $85 \mathrm{kDa}$, but was not affected by the blocking peptide (Fig $\mathbf{1}$, Gel B, Lane 4). Using the porcine specific anti-CD44 monoclonal antibody (Fig 1, Gel C), the CD-44 receptor in pig and human spermatozoa (and the vesicular glands) appeared at 85 $\mathrm{kDa}$ (Lanes 2-4). Co-incubation of the monoclonal primary antibody with its specific blocking peptide (CD44 Fusion Protein Ag7633, Nordic BioSite, Proteintech Europe, Manchester, UK), eliminated the $85 \mathrm{kDa}$ band (Fig 1, Gel D, Lane 3).

The immunocytochemistry (ICC) of porcine spermatozoa depicted the presence of the CD44 receptor on the plasma membrane over the neck and the principal piece irrespectively of the antibody used (Fig 2B+D). In addition, polyclonal antibody in boar sperm leads to an unspecific-like or isoform detection in the tail membrane (Fig 2B) and also over the acrosome. Human spermatozoa had a clear CD44-immunolabelling over the post-acrosome area of the sperm head (Fig $\mathbf{2 A}+\mathbf{C}$ ). Negative controls (inserts) were clearly deprived from immune labelling (Fig 2A-D).

\section{Discussion}

The present study is, to the best of our knowledge, the first to compare different types of antibodies to determine the location of the full-sized HA-CD44 receptor in specific domains of the plasmalemma of boar spermatozoa. In this study, the polyclonal antibody reacted positively with the human spermatozoa (gamete positive control) and even provided in pig spermatozoa a series of expected isoform bands as per the manufacturer data. These were, however, not all specific isoforms since they could not be blocked by the peptide used. The porcine-specific monoclonal anti-CD44 receptor antibody on the other hand, and the use of 
proper positive somatic porcine controls (vesicular glands), confirmed that a full-sized CD44 receptor is present in the membrane of extended and liquid-stored boar ejaculated spermatozoa (Alvarez-Rodriguez et al, 2018).

This study is considered of particular interest as long as the hyaluronan-binding method, commonly used for selection of "sperm maturity" in vitro, results in the selection of a subpopulation of spermatozoa with intact chromatin (Yagci et al., 2010), but no direct link with their morphology (Petersen et al. 2010). The bimodality obtained in some parameters at physiological and supra- physiological doses of Hyaluronic Acid, could be due to selective blocking of the CD44 receptor (Alvarez-Rodríguez et al. 2018), highlighted by an increase in viscosity and/or accumulation of solutes, particularly when extracellular ice is forming during the dehydration of the sperm cells. Whether these results are associated with different isoforms of the CD44 remains to be explored. Thus, the choice of and inappropriate primary antibody, could lead to a misinterpretation of the results.

In conclusion, a note of caution must be made when using commercially available antibodies particularly those not specifically raised for porcine for assessment of sperm membrane receptors. Validation of the appropriate/correct antibody including use of blocking peptide immunogens in the protocols is mandatory. Further studies in other species should be performed in order to have a prospective validation and detection of presence of these CD44 receptors.

\section{Author contribution}

MAR and MLB performed the experiments and wrote the first draft of the manuscript. HR-M designed the experiments, supervised the work, and corrected the manuscript. MAR and HRM secured funding. All authors approved the final version of the manuscript. 


\section{Acknowledgements}

We would like to thank Alejandro Vicente-Carrillo for his helping in the initial test of the polyclonal anti-CD44 antibody and Stefan Koch and Celia Escudero-Hernandez for their assistance in the WB analysis. The study was made possible by grants from the Swedish Research Council FORMAS (Grant 2017-00946), Stockholm; FORSS (Forskningsrådet i Sydöstra Sverige, Grant 473121 and Grant 745971) and Lions Forskningsfond 2016-postdoc (DNR LIU-2016-00641), Linköping, Sweden.

\section{Conflict of interest}

The authors declare no conflict of interest. 


\section{References}

Álvarez-Rodriguez M, Vicente-Carrillo A, Rodriguez-Martinez H, 2018: Hyaluronan improves neither the long-term storage nor the cryosurvival of liquid-stored CD44-bearing AI boar spermatozoa. J Reprod Dev 64, 351-360.

Castellà Fernández, E. Estudio de la expresión de CD44 en procesos preneoplásicos y neoplásicos de esófago, estómago y páncreas. Doctoral Thesis 2000; ISBN846890036. http://hdl.handle.net/10803/4227.

Day A J, Prestwich G D, 2002: Hyaluronan-binding proteins: tying up the giant. J Biol Chem 277, 4585-4588.

Petersen C G, Massaro F C, Mauri A L, Oliveira J B A, Baruffi R L R, Franco J G, 2010: Efficacy of hyaluronic acid binding assay in selecting motile spermatozoa with normal morphology at high magnification. Reprod Biol Endocrinol 8, 149.

Rodriguez-Martinez H, Tienthai P, Atikuzzaman M, Vicente-Carrillo A, Rubér M, AlvarezRodriguez M, 2016: The ubiquitous hyaluronan: Functionally implicated in the oviduct?. Theriogenology 86, 182-186.

Thorne R F, Legg J W, Isacke C M, 2004: The role of the CD44 transmembrane and cytoplasmic domains in co-ordinating adhesive and signalling events. J Cell Sci 117, 373380 .

Tienthai P, Kimura N, Heldin P, Sato E, Rodriguez-Martinez H, 2003: Expression of hyaluronan synthase-3 in porcine oviducal epithelium during oestrus. Reprod Fertil Dev $15,99-105$.

Tienthai P, 2015: The porcine sperm reservoir in relation to the function of hyaluronan. $\mathrm{J}$ Reprod Dev 61, 245-250.

Toole B P, 2004: Hyaluronan: from extracellular glue to pericellular cue. Nat Rev Cancer 4, $528-539$.

Yagci A, Murk W, Stronk J, Huszar G, 2010: Spermatozoa bound to solid state hyaluronic acid show chromatin structure with high DNA chain integrity: an acridine orange fluorescence study. J Androl 31, 566-572. 
Yokoo M, Tientha P, Kimura N, Niwa K, Sato E, Rodriguez-Martinez H, 2002: Localisation of the hyaluronan receptor CD44 in porcine cumulus cells during in vivo and in vitro maturation. Zygote 10, 317-326. 


\section{Figure legends}

Figure 1. A-D. Analysis of the presence of the CD44 receptor by Western blotting (WB) in human and pig spermatozoa, and vesicular glands. In A, anti-CD44 polyclonal antibody (antiCD44 antibody ab24504; Rabbit polyclonal to CD44; Abcam, Cambridge, UK), the CD-44 receptor was detected in pig and human spermatozoa at $85 \mathrm{kDa}$. Gel $\mathbf{B}$ depicts co-incubation of this polyclonal primary antibody with its specific blocking peptide (ab52122 Human CD44 peptide; Abcam, Cambridge, UK), which eliminated a $25 \mathrm{kDa}$ band of the full-sized CD-44 receptor only in human sperm (arrow). Gel $\mathbf{C}$, using a porcine-specific anti-CD44 monoclonal antibody (60224-1-Ig, Nordic BioSite, Proteintech), the CD-44 receptor was detected in pig and human spermatozoa at $85 \mathrm{kDa}$. Gel D. depicts co-incubation of the monoclonal primary antibody with its specific blocking peptide (CD44 Fusion Protein Ag7633, Nordic BioSite, Proteintech Europe, Manchester, UK), which eliminated the $85 \mathrm{kDa}$ band. L1: ladder, L2: human, L3: boar spermatozoa, L4: boar vesicular glands (somatic positive control).

Figure 2. A-D. Immunolocalization of the CD44 receptor by immunocytochemistry (ICC) analysis in human (A) and pig (B) spermatozoa using a polyclonal antibody (anti-CD44 antibody ab24504; Rabbit polyclonal to CD44; Abcam, Cambridge, UK) and in human (C) and pig (D) spermatozoa using a monoclonal antibody (anti-CD44 antibody 60224-1-Ig; mouse monoclonal antibody to CD44; species specificity: pig; Nordic BioSite, Proteintech Europe, Manchester, UK). The insets in the bottom-left corner in each figure depict ICC negative control images (where the antibody was neutralized by treatment with the specific blocking peptide: for polyclonal (ab52122 Human CD44 peptide; Abcam, Cambridge, UK) and for monoclonal (CD44 fusion protein Ag7633 (Nordic BioSite, Proteintech Europe, Manchester, UK). Human spermatozoa (positive control) had CD44-immunostaining over the post-acrosome area of the sperm head (Fig 2A-C). In pig spermatozoa (Fig 2B-D), the CD44 receptor was localized on the plasma membrane over the post-acrosomal region, neck, and midpiece. Confocal laser scanning microscopy, scale bar: $10 \mu \mathrm{m}$. 